CHAPTER 9

\title{
How to Write a Systematic Review Article and Meta-Analysis
}

\author{
Lenka Čablová, Richard Pates, Michal Miovský and \\ Jonathan Noel
}

\section{Introduction}

In science, a review article refers to work that provides a comprehensive and systematic summary of results available in a given field while making it possible to see the topic under consideration from a new perspective. Drawing on recent studies by other researchers, the authors of a review article make a critical analysis and summarize, appraise, and classify available data to offer a synthesis of the latest research in a specific subject area, ultimately arriving at new cumulative conclusions. According to Baumeister and Leary (1997), the goal of such synthesis may include (a) theory development, (b) theory evaluation, (c) a survey of the state of knowledge on a particular topic, (d) problem identification, and (e) provision of a historical account of the development of theory and research on a particular topic. A review can also be useful in science and practical life for many other reasons, such as in policy making (Bero \& Jadad, 1997). Review articles have become necessary to advance addiction science, but providing a systematic summary of existing evidence while coming up with new ideas and pointing out the unique contribution of the work may pose the greatest challenge for inexperienced authors.

\section{How to cite this book chapter:}

Čablová, L, Pates, R, Miovský, M and Noel, J. 2017. How to Write a Systematic Review Article and Meta-Analysis. In: Babor, T F, Stenius, K, Pates, R, Miovský, M, O'Reilly, J and Candon, P. (eds.) Publishing Addiction Science: A Guide for the Perplexed, Pp. 173-189. London: Ubiquity Press. DOI: https://doi.org/10.5334/bbd.i. License: CC-BY 4.0. 


\section{What is the Relevance of a Review?}

General definitions are one thing; the practical benefit of writing reviews is another. Why would a novice author/researcher engage in this activity? Why is it important? What benefits can it bring? First, it provides the authors with a general understanding of the subject matter they study as part of their area of expertise. Each field of study has its own terminology, and the more specific a topic is, the greater the terminological differences that may be found among authors. It is therefore important to produce a good description and critical appraisal of existing evidence concerning the topic being explored. Another objective is to integrate the findings generated by different studies into a meaningful body of evidence. The process of writing a review article will help the authors obtain a unique perspective on the issue and assist them in processing the results from many investigators into a consistent form. It will then be possible to summarize the results and interpret the existing evidence in a new light. To increase one's chances of having a review article accepted for publication, it is useful to address topical issues in a given field or areas of research featuring a number of heterogeneous and controversial studies where a consistent approach is needed.

\section{What is a Review?}

It is difficult to provide a single definition of a review. Indeed, each journal uses its own-slightly different-definition of a review study. For example, the journal Adiktologie defines a review article as a "cogent summary of topical issues; the author's own experience is not the underlying theme of the paper. The maximum extent is 16 pages, with not more than 50 bibliographical citations. References to recent literature (not more than five years old) should prevail" (Gabrhelík, 2013). Addiction, meanwhile, simply states that "reviews draw together a body of literature to reach one or more major conclusions" and allows review articles to contain up to 4,000 words with no limit on bibliographic citations (Society for the Study of Addiction, 2015).

Despite these limitations, clear distinctions can be made between the types of reviews that can be drafted. The traditional type of review is a narrative literature review, which assesses the quality and results of a selection of literature using implicit criteria (Culyer, 2014). The conclusions of traditional narrative reviews are often based on subjective interpretations of the literature and may be biased in unsystematic ways. Importantly, narrative reviews are essentially nonreplicable.

In contrast, scientific journals often require reviews to be systematic in nature. Systematic reviews use explicit literature search strategies, inclusion and exclusion criteria, and criteria for determining the quality and reliability of study findings. Systematic reviews are replicable and the conclusions drawn by authors more easily verified. 
A systematic review that does not include an evaluation of study findings (i.e. performs only a systematic search using explicit inclusion and exclusion criteria) is referred to in this chapter as a hybrid narrative review. Hybrid narrative reviews provide authors greater freedom to interpret and integrate study results and conclusions compared with systematic reviews but still allow the reader to determine the authenticity of the author's findings. These reviews are particularly important for theory development and problem identification, especially when the peer-reviewed literature may be incomplete and when important studies may not use rigorous experimental or longitudinal designs.

Meta-analyses are a step beyond systematic reviews; they require a quantitative analysis of previously published findings.

The following sections discuss the steps involved in creating systematic reviews and meta-analyses. Although not explicitly mentioned, much of the information applies to hybrid narrative reviews as well. Because traditional narrative reviews are no longer viewed favorably, they will not be discussed. It is strongly recommended, however, that before writing any article, authors should first choose a journal to which to submit their research because of the subtle differences in journal manuscript definitions. Authors should study thoroughly the guidelines for authors and keep them on hand to reference while writing the article. This may save a great deal of time spent on final revisions or even make them unnecessary.

\section{Main Steps to Successful Systematic Review}

It is useful to observe the following procedure when designing and writing a systematic review. If the intention is to arrive at a systematic classification of evidence, a well-considered and highly structured procedure should be used. Structure is a crucial requirement, and some specific tools (e.g., PICOS: participants, interventions, comparators, outcomes, and study design) can make this more manageable (Smith et al., 2011). Below, we describe the specific steps involved in creating a systematic review and meta-analysis, using the development of a previously published review as an example of good practice. The following recommended strategies are based on the published systematic review (Čablová et al., 2014).

\section{Aim of the Review}

The aim of a systematic review is set in the same way as in an original research study; the article must contribute something new to the given research field. The specific aim should correspond with the research questions. It may be, for example, "to provide a systematic review of the results of studies published from 2000 to 2012 that investigate the specific relationship between the level 
of parental control and alcohol use among children and adolescents." Alternatively, it may be "to classify parenting strategies in relation to alcohol-using children aged 12-15" or "to make a critical appraisal of recent studies of the emotional bond in young adults who use cannabis."

The aims are typically stated in the last paragraph of the introduction. The aims then determine the choice of the specific procedure used to search sources and process and present the results. In the concluding section of the study, it should be stated whether and to what extent the aims have been fulfilled.

\section{Inclusion of Research Questions}

In a review article, the research question is included and expressed in the text, formulated as the problem: the topic and the focus of the work. It can be thought of as a spiral that provides logical connections among the parts of the article; that is, different parts build on and follow up on each other in a logical pattern. In terms of a systematic review, the research question must correspond with the objectives of the study and be aligned with the methodology, which is particularly relevant for the identification of data sources (the literature search) and the determination of study inclusion and exclusion criteria. It represents an imaginary starting point for the selection of key words and other parameters that are looked for in the relevant studies. As an example, we can use an article investigating the quality and type of emotional bonds in young adults who use cannabis and its (implicit) research question: "Can an insecure emotional bond be associated with a higher rate of cannabis use among young adults?" or: "Is there a relationship and difference between the lifetime prevalence of cannabis use among young adults and the individual types of insecure emotional bond?"

\section{Identify Data Sources-Quality Literature Search}

The primary and most important data sources are electronic databases, typically accessed through university libraries. Because access to specific papers may be limited as a result of financial constrictions, the levels of access granted to students and staff will depend on the resources of the university subscribing to the journals. Thus, you may find that although you can get into a number of databases, you may be able to access only a few full texts (as the others require payment) and have mostly abstracts available, which may not be sufficient for systematic reviews. This is dealt with in more detail in the next point.

In the field of addictology, we recommend to use following databases:

- Web of Science: http://www.webofknowledge.com

- Medline/PubMed: http://www.ncbi.nlm.nih.gov/pubmed

- EBSCO: http://search.ebscohost.com 
- SCOPUS: http://www.scopus.com

- ProQuest Central: http://search.proquest.com/index

- PsycARTICLES: http://www.apa.org/pubs/databases/psycarticles/index. aspx

Nevertheless, databases and full-text studies are not the only data sources. It is also possible to include conference presentations if the conference abstracts have been published. At the same time, some journals could have a problem with these types of publications because they did not undergo a standard peer-review process. Also, a quality literature search should not disregard print sources, such as monographs; articles in peer-reviewed, non-indexed journals; handbooks and manuals pertaining to the relevant topic; graduate theses; and dissertations. These could be included into a category "Records identified through other sources" in the PRISMA (Preferred Reporting Items for Systematic Reviews and Meta-Analyses) study flow diagram (see below).

We recommend keeping scrupulous notes on the articles read, either using Endnote or a separate database of references. This is relevant to all research but particularly to reviews.

\section{Determine Selection Criteria}

The relevant publications, the results of which are to be processed, are selected according to the classification criteria that follow.

- Year of publication-designating the period that is under study-may be used as the first criterion.

- Number of citations of the article-this information can be found in databases, most often under the heading "Times cited." Articles with a greater number of citations report on more prestigious research.

- Key words - they reflect the terminology used in the given field and also help identify the most relevant studies.

- Relevance of the article-online databases may turn up a number of articles but, unfortunately, because of the potential overlap of key words and other parameters, some works may be totally inconsistent with the focus of the review. It is therefore necessary to look through each publication-in most cases the abstract will be enough-and exclude any irrelevant studies.

- Type of publications - although you may typically work with original and review studies only, specific topics may require the use of information from annual reports, research reports, or guidelines. It is therefore important to state these factors in the description of the procedure.

- Study design - as far as research studies are concerned, these may be further divided into subcategories: for example, reviews versus original works or, with clinical issues in particular, cross-sectional versus longitudinal. 
- Language of the publications - the languages that currently predominate in science are English and Spanish, with Chinese emerging as a significant language of science (in addition to English, Web of Science databases provide the option of searching studies in Chinese).

- Sociodemographic environment-it is useful to describe the sociodemographic environment in which the research was conducted because it is a relevant factor that may influence the review's results. Thus, the review needs to take this into account when presenting the research results.

- Funding source and conflicts of interest-last but not least, the funding source of a study and other conflicts of interest may influence how the results are interpreted. As explained in other chapters, significant biases in study reporting have been uncovered when the funding source or authors have a financial stake in the results of the study.

Entered into a database or observed when working with hard-copy sources, these criteria make it possible to focus the work on the research question and the aim of the study you have laid down. Finally, all these criteria/indicators will be considered and interpreted in the subsequent discussion section.

\section{Process of "Data Collection"}

The complete literature search process needs to be recorded and documented. When evaluating systematic reviews, peer reviewers pay special attention to the means used to collect the "data" (i.e., specific publications) for the analysis. There are specific methods that can be applied for this purpose, with the PRISMA study flow diagram being the most frequently used one in contemporary science (Higgins \& Green, 2008; Moher et al., 2009). Figure 9.1 shows the PRISMA study flow diagram used in the systematic review (Čablová et al., 2014).

\section{Explanation of the Specific Items in the Prisma Study Flow Diagram}

The first item, Records identified through database searching, shows the number of publications found in databases on the basis of the selection criteria. The item Additional records identified through other sources refers to the number of publications found in information sources other than those available online (these are typically print documents, such as research reports, handbooks, and manuals). Another step involves the elimination of duplicate articles. If you work with multiple databases, it is very likely that the same publication will be selected several times. Such duplicates should therefore be removed. This process is very easy if you use a citation manager. When using EndNote, for example, this can be achieved by simply activating the "Find duplicates" function. 


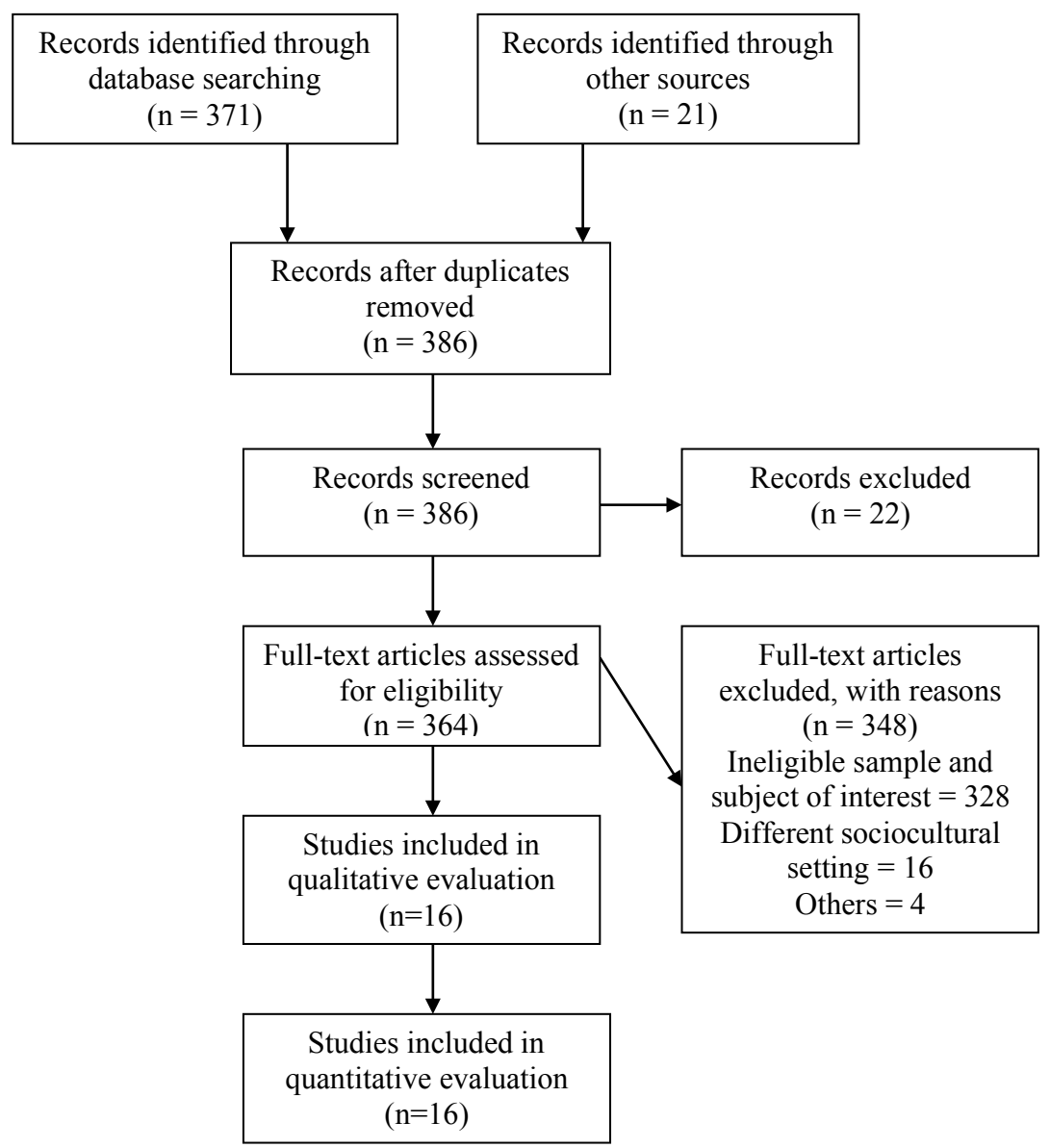

Figure 9.1: PRISMA study flow diagram.

Source: Čablová et al. (2014, p. 4).

Then you can focus on the articles. The item Records screened indicates the number of publications that remained after the exclusion of duplicates and publications rejected after you have read the abstracts. The number of articles eliminated on the basis of the examination of their abstracts is indicated in the Records excluded box. On the other hand, articles for which the full text is available (these should make up as large a proportion of the initial set of records as possible) are assessed in the next step and their final number is given under Full-text articles assessed for eligibility. When reading through the studies, you should continue to bear in mind the selection criteria (ideally, with a checklist on your desk) and watch carefully for them being met in the studies under scrutiny. If a more rigorous design is applied, you can also create a table 
specifically for the selection and assessment of publications. If you come across articles that do not meet the selection criteria, you should state the reasons for such ineligibility and the respective number of studies; see the item Full-text articles excluded with reasons. The last figure shows the final number of articles included in the study. This example contains two alternatives-Studies included in qualitative evaluation and Studies included in quantitative evaluation-but one item only, for example, Studies included in quantitative evaluation, is also possible. For more information about the PRISMA study flow diagram method, including further illustrations of the procedure or the PRISMA checklist that helps in keeping a record of the process, visit http://www.prisma-statement. org/statement.htm.

\section{Interpretation of Results}

The results of the studies you have obtained will be further summarized in a structured form-ideally a table-according to the classification criteria. It is advisable to compare the qualitative and quantitative perspectives of the studies when processing the results. (Although meta-analysis is not always the goal, it is useful to take quantitative as well as qualitative approaches into account.) When using a quantitative point of view, you can follow the number of studies that used a longitudinal versus cross-sectional design, how many studies applied a standardized methodology versus a methodology developed specifically for the purposes of the study, or how many studies had their samples of participants well balanced in terms of representativeness and how many did not. On the other hand, a qualitative perspective makes it possible to look for broader aspects of the works and fine subtleties in the results that have been ascertained.

There are a number of available tools that can serve as a guide when examining study methodologies and results. The Consolidated Standards of Reporting Trials (CONSORT) statement provides a standardized way to report and interpret the results of randomized clinical trials (Schulz et al., 2010). The primary tool is a 25 -item checklist that contains questions on how the trial was designed, the data analyzed, and the results interpreted. The Strengthening the Reporting of Observational studies in Epidemiology (STROBE) and Transparent Reporting of Evaluations with Nonrandomized Designs (TREND) statements are similar checklists for studies using observational study designs (von Elm et al., 2007; des Jarlais et al., 2004). If a more quantitative analysis of study design is desired, the recommendations of the Grades of Recommendation, Assessment, Development, and Evaluation (GRADE) working group may be used (Atkins et al., 2004). These recommendations contain a point system that can be used in combination with the CONSORT, STROBE, or TREND statements to further differentiate among studies. Although useful, the results of using these tools should not be considered as absolute but as guides toward 
determining the weight that a study's conclusions should be given. In addition, systematic reviews should always be attentive to sex and gender issues, as described in the SAGER Guidelines (Heidari et al., 2016).

Interpretation should always be based on the results and findings specified in a given study; you must refrain from adding any conclusions of your own, because the principal rule is to preserve and express the original author's idea as precisely as possible. When formulating the ideas and working with other review studies, you should always look up the primary source and interpret its results. Other review studies may serve as an inspiration in classifying your results rather than being their source, functioning rather as "background material."

Any copyright rules should be observed when making citations. You should strictly avoid using findings presented by the original authors in their research as your interpretations; if at all, you can resort to a secondary citation, which in itself may appear rather awkward. Therefore, you should seek to be as accurate as possible and restate the author's original argument, looking up other relevant works on the topic that you will cite in the same way. In addition, it is necessary to be attentive and socially sensitive when interpreting the results of studies from different sociocultural settings; you should be careful not to make unreasonable generalizations and ensure that the results are always interpreted in terms of the given social context. This may involve engaging in some additional research but, particularly in the social science field, this extra effort is an element that has a major impact on the final product. In Table 9.1 we present an example that illustrates the processing of the results in a published systematic review (Čablová et al., 2014). The left hand column lists the studies according to authors and year, which corresponds with the standard identification of citations in text. The selection criteria applied to the studies under consideration are indicated in the heading line. The reader thus has a chance to see the results of the work in aggregate and in a clearly structured way without having to wade through a lot of text.

\section{Discussion and Conclusion-was the Aim Really Achieved?}

Once the results have been processed and interpreted, what is probably the most challenging part comes next. For one thing, you may be quite tired by now, because the previous systematic procedure was rather demanding in terms of attention and endurance, and now you need to think about the results and compare them with the conclusions drawn by other relevant studies and with each other. In particular, this requires you to bring a new perspective to the subject matter under study, singling out and discussing most salient finding from the results. Importantly, the discussion should compare and evaluate the results against other relevant research projects rather than against the presentation of the author's opinions on the issue. Each idea or result presented in the 


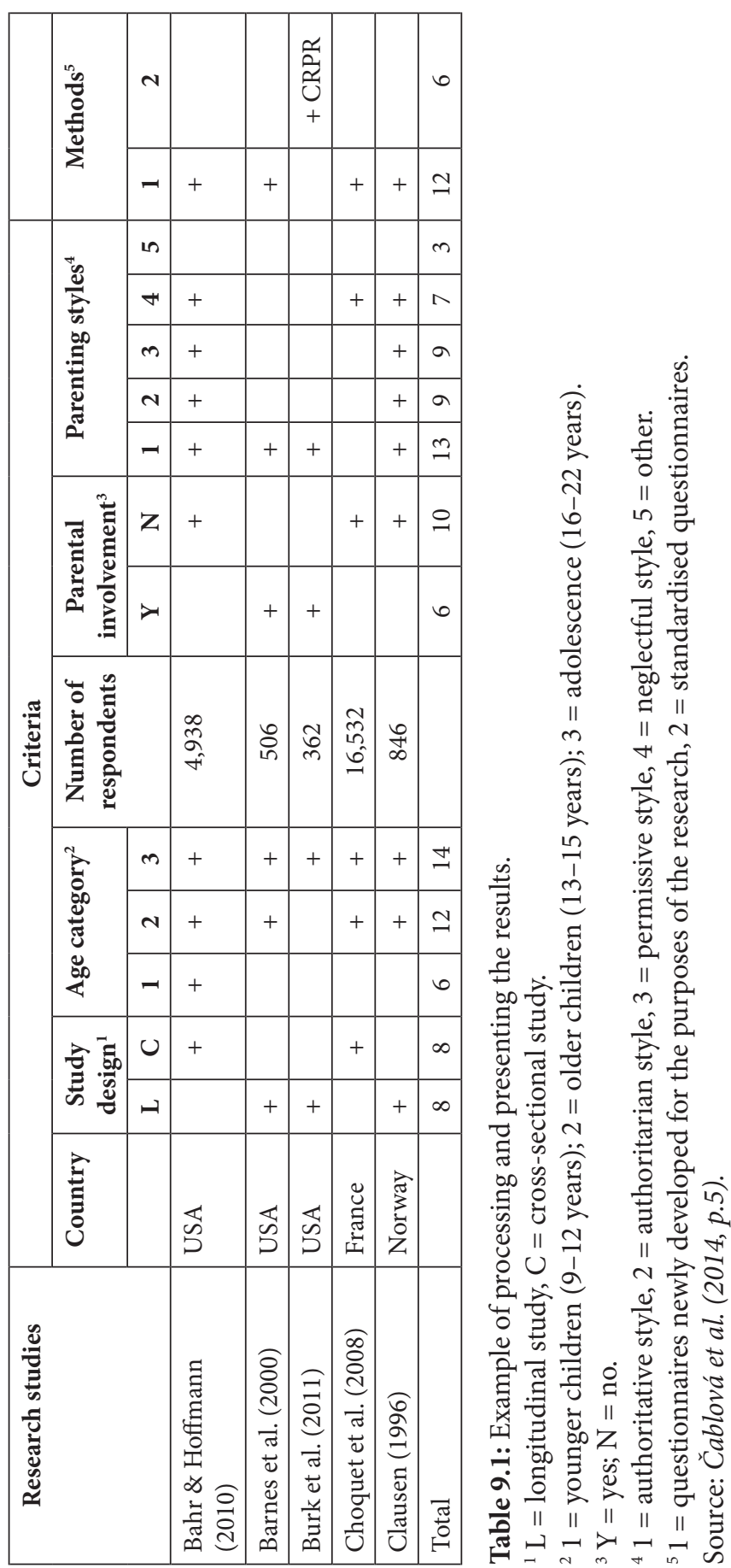


article needs to be properly cited, too. The conclusion consists of a practical evaluation of the study; it should not contain any new findings or evidence. Its purpose is briefly to summarize the results and the contribution of the study as a whole. Although this can pose a formidable task to an inexperienced author, it is important to practice the skill of communicating your own views concisely.

The conclusion often includes recommendations (resulting from the study) for further research and tips for practice. It is also advisable to highlight the unique contributions of your review. In technical terms, it is recommended to study carefully the instructions for authors provided by the journal in which you want to submit the article for publication. Although some journals require the discussion and conclusion to come in two separate sections, others prefer to have them combined. The latter requires a slightly different structure, and it is helpful to be familiar with the format requirements before writing the article.

\section{The Most Frequent Pitfalls}

When trying to pursue as systematic and transparent a procedure as possible, you can encounter several problems. We have already mentioned the potential problem with differences in terminology used by the authors who publish research on a given subject in the field. To prevent confusion, it is recommended that you read a reasonable number of articles pertaining to your topic and look for the terminology they use. Databases may be helpful in this. The Web of Science platform, for example, features a "related records" function, which may be used to search for similar articles on a certain topic. You may be confronted with a range of often competing theoretical approaches or backgrounds used by the authors to explore the subject matter in question. Because the literature search may be a challenging and time-consuming task, you may need to allow some time to study the relevant concepts thoroughly (for which the studies you have identified may not provide all the answers, requiring you to do further reading), as well as to reflect on such differences in your own conclusions and interpretations. Other differences may be found in the methodology applied by the studies under scrutiny. There are authors who work with standardized methods and their results can be subjected to a simple and valid comparison; on the other hand, there are authors who use their own methodology and whose results are thus difficult to measure. Another aspect that will consume time is the elimination of duplicate records, because researchers sometimes publish the results of the same study in several parts, divided into various subtopics to meet the foci of different journals. A mechanical "remove duplicates" function cannot do all the work. It is necessary to be alert and watch out for any relevant correlates.

Another problem that may be encountered when comparing results between studies is the difference in the number of study participants. Many studies do not use a representative sample of participants, and great differences in their 
sizes may strongly affect study generalizability. You may also face your own limitations, particularly regarding the inclination toward a selective choice of studies, where certain studies may not be included, either deliberately or inadvertently. Because citation bias may significantly compromise the results, you should try to avoid it at all costs if you want to arrive at a conclusion that is relevant to the field. If you fail to do so, it is most likely that reviewers will discover such a bias, as it is their job to examine related studies in the given area of research.

The last aspect to consider during the interpretation process is the statistical versus clinical significance of studies. In a large number of cases, you will find results that are not reflected in clinical practice, despite being significant. Therefore, it is important to maintain contact with clinical practitioners (or consult other experts) and be able to compare the results with real life. You can then formulate how these significances correlate in the conclusion.

For addiction science, the critical evaluation of systematic reviews is quite important. It is the key to the correct interpretation of selective data from particular studies, it provides background for comparing findings, and it can help to identify potentially disproportionate or inhomogeneous interpretations of findings. It has always been a sensitive issue in the context of publishing addiction science because of potential conflicts of interest, and the history of the field contains examples of published papers in which researchers intentionally distorted data. The tendency to interpret data in a different way and present specific points of view can be a potential source of bias (Bero \& Jadad, 1997). For example, there are many examples of contrasting study findings in the area of tobacco policy depending on whether the study was or was not sponsored by the tobacco industry (Glantz, 2005).

\section{Meta-Analysis}

Meta-analysis is a form of systematic review that combines findings from a number of studies to create aggregate effect sizes. To do this, the size of the effect is calculated and indexed. This can be used for a number of purposes in addiction science, including the effects of an intervention (e.g., the use of naltrexone and acamprosate for treating alcohol use disorders [Maisel et al., 2013] or the impact of smoking bans on restaurants and bars [Cornelson et al., 2014]) and epidemiology (e.g., substance use among street children [Embleton et al., 2013]) or seroconversion of hepatitis $C$ in relation to shared syringes [Pouget et al., 2012]). By aggregating the effects and applying a statistical analysis, a better understanding may be obtained for some of these research questions.

This is a complicated and time consuming process, probably not best undertaken by inexperienced researchers, but it may add greatly to the better understanding of science and aid treatment providers and policy makers. The process is not dissimilar to that described above in terms of selecting articles 
for systematic reviews but requires a more complicated analysis. There are also similarities with primary intervention trials, in which one focuses on how well an intervention works. However, in a meta-analysis, the researcher looks across studies to determine the magnitude of effects. It is worth following a systematic guideline such as PRISMA to establish a framework for the review (Moher et al., 2009).

The first step is to formulate the research question. Decide the keywords you will use to search for articles, the date from which you wish articles to be included, and the inclusion and exclusion criteria. Search the databases you have chosen for articles that meet your subject and eligibility criteria. It is also worth looking at reference lists from the articles you have selected to find other articles not so far identified.

Once the articles for inclusion have been identified they will need to be coded according to the variables chosen for the meta-analysis. Because these coding decisions are not always clear, two raters are often used to obtain some measure of reliability either by percent agreement or by a kappa coefficient. Enter the data extracted onto a database with relevant details of each study entered including, for example, type of intervention, follow-up periods, sample size, type of control group, and research design.

One of the problems in comparing a number of studies is that studies will report diverse outcomes according to the model they used. To determine effect sizes so that the meta-analysis is effective, a "common currency" of effects needs to be established in order for comparisons and aggregation to be made. Finney and Moyer (2010) suggest that the most common effect sizes used are standardized mean difference, odds ratio, and correlation coefficient. The standardized mean difference is "the difference between means on a continuous outcome variable for an intervention and a comparison condition, typically divided by the pooled standard deviation of the two groups." (Finney and Moyer, 2010, pp 321). By using standard deviations, one can measure by how many standard deviations, or what proportion of standard deviations, the intervention is performing better than the control group.

Another method of measuring effect size is by using the odds ratio. By calculating the probability of something changing divided by something not changing, a ratio may be obtained. An odds ratio of 1.00 would show that there was no difference between treatment and a control condition in which there were two possible outcomes.

The third method is the correlation coefficient, which can be used to express the relationship between a continuous intervention dimension (which is unusual in addiction studies) and the outcome (Finney \& Moyer, 2010).

We have now established a method of calculating effect sizes, and, to find out whether there is indeed an effect and what that effect is, we must now aggregate them across the studies we have reviewed. This can be done with a fixed-effects or a random-effects approach. These two approaches deal with the study sampling errors, with the former assuming that the error in estimating 
the population effect size comes from random factors associated with subjectlevel sampling, whereas the latter assumes that there are study sampling errors in addition to subject-level sampling errors. A random-effects model is used more frequently because of a greater generalizability, although the fixed-effects model has a greater statistical power. Effects from larger sample sizes have less variance across studies and are therefore more precise. To test whether the overall effect size varies from zero, it is best to use specific statistical software designed to conduct meta-analyses (Finney \& Moyer, 2010).

As with systematic reviews, a table should be presented detailing all the articles included in the study and describing all the relevant characteristics, including author, date of data collection, the main outcome findings, and methods of collecting the data. A forest plot that shows the range of findings for each study is also often included, detailing in comparison the range of effects in an intervention.

\section{Issues with Meta-Analysis}

There a number of issues that should be considered when conducting a metaanalysis. One may have to determine whether the effect sizes vary more than could be expected from subject-level sampling fluctuations in a fixed-effect model or, in a random-effect model, whether there are study-level random effects in addition to the subject-level sampling fluctuations. Are there additional factors that add variation in effect sizes explained by moderator variables? The moderator variables include different methods and participants across the studies and the interventions themselves. To test this, a homogeneity test can be used that will test for whether excess variation exists (Viechtbauer, 2007).

Another problem is publication bias. If the articles are selected carefully from peer-reviewed journals and conform to the criteria for inclusion, there is still the problem in that studies that show no positive or neutral results are often not published, either because the researchers do not submit for publication or because the papers are rejected for publication. Therefore, any articles that refute the research question may not be included in the databases searched and therefore the results may be skewed.

Selection of the articles needs to be done with great care. Only quantitative articles may be included-qualitative articles will not contribute a statistical outcome-and if the criteria are too strict, then the number of articles on which to base the analysis may be too small. On the other hand, if you the selection criteria are too wide, you may then include studies of poor quality that will affect the outcome of the meta-analysis. The other problem with selection of articles may be agenda bias, whereby the authors of the meta-analysis want to use the results to support a specific issue and may cherry pick the articles they include. Meta-analysis is complicated, and the analysis of the variance across articles is complex; therefore, it is always beneficial to get good statistical advice and to use an established statistical package for analyzing the data. 


\section{Conclusion and Final Advice}

As previously mentioned, a good review article is hardly possible without a good literature search. The literature search has its own rules that generally apply to both original and review studies. A systematic review involves a literature search procedure guided by the principle of keeping an accurate and transparent record of the entire process! It is useful to create a summary Excel table where citations of studies will be recorded according to the selection criteria. It may seem like extra work at the beginning, but the author will come to appreciate this facility even before the first round of the peer-review process is over. Indeed, peer reviewers very easily notice any shortcomings we have tried to hide. It is therefore strongly recommended to draw up and enclose with the article a diagram in which you document the procedure for selecting the studies. This will help reviewers understand the approach and the results obtained, and, if any queries should arise, this evidence will make it easy to refute and explain any misgivings about the process or the results. For these purposes, it is also recommended to archive the documents in both printed and computerized versions; a physical file for hard copies and a separate electronic folder for computerized counterparts may be a useful option, with the latter providing the extra convenience of the "find" functionality.

To summarize, the ultimate goal when developing a review article is a systematic, straightforward, and transparent procedure. Both the reader and the editor must be clear about what the aims and methodology are, and all the results must be in line with the methods used. Although certain variations on standard procedures are possible, they always need to be explained and justified in discussion; otherwise you will most likely deal with them in the first round of the peer-review process. There are some specific approaches and tools for quality assessment of reviews (e.g., AMSTAR [Smith et al., 2011]; MOOSE [Stroup et al., 2000]) that can be relevant and very helpful in determining what is assessed and how to make the manuscript better.

Please visit the website of the International Society of Addiction Journal Editors (ISAJE) at www.isaje.net to access supplementary materials related to this chapter. Materials include additional reading, exercises, examples, PowerPoint presentations, videos, and e-learning lessons.

\section{References}

Atkins, D., Best, D., Briss, P. A., Eccles, M., Falck-Ytter, Y., Flottorp, S., . ., Zaza, S., \& GRADE Working Group. (2004). Grading quality of evidence and strength of recommendations. BMJ, 328, 1490. 
Baumeister, R. F., \& Leary, M. R. (1997). Writing narrative literature reviews. Review of General Psychology, 1, 311-320.

Bero, L. A., \& Jadad, A. (1997). How consumers and policymakers can use systematic reviews for decision making. Annals of Internal Medicine, 127, $37-42$.

Čablová, L., Pazderková, K., \& Miovský, M. (2014). Parenting styles and alcohol use among children and adolescents: A systematic review. Drugs: Education, Prevention, and Policy, 1, 1-13.

Cornelson, L., McGowan, Y., Currie-Murphy, L., \& Normand, C. (2014). Systematic review and meta-analysis of the economic impact of smoking bans in restaurants and bars. Addiction, 109, 720-728.

Culyer, A. J. (2014). The dictionary of health economics (3rd ed.). Cheltenham, England: Edward Elgar Publishing Limited.

Des Jarlais, D. C., Lyles, C., Crepaz, N., \& TREND Group. (2004). Improving the reporting quality of nonrandomized evaluations of behavioral and public health interventions: The TREND statement. American Journal of Public Health, 94, 361-366.

Embleton, L., Mwangi, A., Vreeman, R., Ayuku, D., \& Braitstein, P. (2013). The epidemiology of substance use among street children in resourceconstrained settings: A systematic review and meta-analysis. Addiction, 108, 1722-1733.

Finney, J. W., \& Moyer, A. (2010). Meta-analysis: Summarising findings on addiction intervention effects. In P. G. Miller, J. Strang, \& P. M. Miller (Eds.), Addiction research methods. Oxford, England: Wiley-Blackwell.

Gabrhelík, R. (2013). Guidelines for Authors (updated January 2013). Adiktologie. Retrieved from http://www.adiktologie.cz/en/articles/ detail/459/4021/GUIDELINES-FOR-AUTHORS.

Glantz, S. (2005, September). Why you should not publish any tobacco funded research. Presented at the annual ISAJE conference, San Francisco, CA.

Heidari S., Babor T. F., De Castro P., Tort S., \& Curno M. (2016). Sex and Gender Equity in Research: Rationale for the SAGER guidelines and recommended use. Research Integrity and Peer Review, 1:2. DOI: https://doi.org/10.1186/ s41073-016-0007-6

Higgins, J. P. T., \& Green, S. (Eds.). (2008). Cochrane handbook for systematic reviews of interventions (Version 5.0.1 [updated September 2008]). The Cochrane Collaboration. Retrieved from www.cochrane-handbook.org.

Maisel, N. C., Blodgett, J. C., Wilbourne, P. L., Humphreys, K., \& Finney, J. W. (2013). Meta-analysis of naltrexone and acamprosate for treating alcohol use disorders: When are these medications most helpful? Addiction, 108, 275-293.

Moher, M., Liberati, A., Tetzlaff, J., Altman, D. G., \& The PRISMA Group. (2009). Preferred reporting items for systematic reviews and meta-analysis: The PRISMA statement. Journal of Clinical Epidemiology, 62, 1006-1012. 
Pouget, E. R., Hagan, H., \& Des Jarlais, D. (2012). Meta-analysis of hepatitis C seroconversion in relation to shared syringes and drug preparation equipment. Addiction, 107, 1057-1065.

Schulz, K. F., Altman, D. G., Moher, D., \& CONSORT Group. (2010). CONSORT 2010 statement: Updated guidelines for reporting parallel group randomised trials. PLoS Medicine, 7, e1000251.

Smith, V., Devane, D., Begley, C. M., \& Clarke, M. (2011). Methodology in conducting a systematic reviews of healthcare interventions. BMC Medical Research Methodology, 11, 15.

Society for the Study of Addiction (2015). Instructions for Authors. Addiction. Retrieved from http://www.addictionjournal.org/pages/authors (August 11, 2015 ).

Stroup, D. F., Berlin, J. A., Morton, S. C., Olkin, I., Williamson, G. D., Rennie, D., . ., \& Thacker, S. B. (2000). Meta-analysis of observational studies in epidemiology: A proposal for reporting. Meta-analysis of Observational Studies in Epidemiology (MOOSE) group. JAMA, 283, 2008-2012.

Viechtbauer, W. (2007). Hypothesis testing for population heterogeneity in meta-analysis. British Journal of Mathematical and Statistical Psychology, 60, 64-75.

von Elm, E., Altman, D. G., Egger, M., Pocock, S. J., Gøtzsche, P. C., Vandenbroucke, J. P., \& STROBE Initiative. (2007). The Strengthening the Reporting of Observational Studies in Epidemiology (STROBE) statement: Guidelines for reporting observational studies. PloS Medicine, 4, e296. 
